\title{
Sonar guided focused parathyroidectomy under cervical block
}

\author{
I Bombil ${ }^{1}$, L Louw ${ }^{2}$, C Mitchell ${ }^{1}$, F Mahlobo ${ }^{3}$, RA Muganza ${ }^{1}$, NR Madima ${ }^{4}$ \\ ${ }^{1}$ Department of Surgery, \\ ${ }^{2}$ Department of Nuclear Medicine \\ ${ }^{3}$ Department of Radiology \\ ${ }^{4}$ Department of Anesthesiology. \\ Chris Hani Baragwanath Academic Hospital and the Faculty of Health sciences. University of Witwatersrand, Johannesburg, \\ South Africa
}

Corresponding author: I Bombil (bombil@telkomsa.net)

\begin{abstract}
Introduction: Presentation of hyperparathyroidism varies and is highly non-specific.
The automated calcium analyzer has made the diagnosis easy. Similarly, the advent of Sestamibi scan has paved the way to minimally invasive parathyroidectomy indicated for parathyroid adenoma. There is no uniformity in the extent of minimally invasive parathyroidectomy that is done through limited incision under radio or sonar guidance and endoscopically. In this study, we are presenting the focused parathyroidectomy performed under sonar guidance and superficial cervical block (SCB). The prerequisite is concordant preoperative Sestamibi and ultrasound imaging.

Objective: To reflect on the result of sonar-guided focused parathyroidectomy under SCB.

Method: A two-year review of parathyroidectomies performed between January 2013 and December 2014.

Result: There was good correlation between the pre-operative imaging, the intra-operative findings and the postoperative histology result of the 15 cases analysed.

Conclusion: The focused parathyroidectomy under SCB yielded a good result with concordant preoperative Sestamibi and ultrasound findings.
\end{abstract}

\section{Introduction}

Presentations of hyperparathyroidism are unpredictable. Signs and symptoms of hypercalcaemia are non-specific and are compounded by the manifestations of the affected target organs. There are various sources of referral to endocrine unit and eventually to surgery from different medical subspecialties in keeping with the ambiguity of the clinical pictures. In the developed world, the routine use of automated calcium analyzer has led to early detection of hypercalcaemia in patients with subtle or no obvious symptoms. ${ }^{1}$

Before the advent of ${ }^{99 \mathrm{~m}} \mathrm{Tc}-\mathrm{Sestamibi}$, the best management of parathyroid adenoma was four-gland exploration without prior localization. ${ }^{1}$ Other imaging modalities (CT scan, MRI, sonar) were neither sensitive enough nor specific enough to be relied upon and were reserved for redo cases only. ${ }^{1}$ Imaging with ${ }^{99 \mathrm{~m}} \mathrm{Tc}$-Sestamibi has been the technique of choice for parathyroid localization for over two decades and has largely replaced subtraction imaging with ${ }^{201}$ Thallium. $^{2}$

${ }^{99 \mathrm{~m}} \mathrm{Tc}-\mathrm{Sestamibi}$ is highly sensitive $(90.7 \%)$ and specific $(98.8 \%)$ in the identification of parathyroid adenoma. ${ }^{1}$ ${ }^{99 \mathrm{~m}} \mathrm{Tc}-\mathrm{Sestamibi}$ has revolutionised the approach to primary hyperparathyroidism. It is now possible to perform minimal access surgery of the parathyroid with very good results. ${ }^{3-5}$ Ever since, there has been a rise of minimally invasive parathyroidectomy (MIP) done via a small incision under radio, ultrasound guidance or endoscopically. ${ }^{6-11}$ The MIP is limited to primary hyperparathyroidism, more specifically to parathyroid adenoma. In sporadic parathyroid adenoma, Denham and Norman noted the incidence of single adenoma, four glands hyperplasia, multiple adenomas and cancer to be $87 \%, 9 \%, 3 \%$ and less than $1 \%$ respectively. ${ }^{1}$ Based on this finding, a large number of four gland explorations need to be performed to identify rare occurrence of multiple adenomas. Failing to localise any parathyroid in the presence of biochemical evidence of primary hyperparathyroidism may indicate hyperplasia in which case minimal access is contraindicated. The term "MIP" may be subjected to different interpretation, as we cannot ascertain what length of incision and extent of dissection represent MIP. We prefer the term "focused parathyroidectomy" to refer to the small incision (usually $2.5 \mathrm{~cm}$ ) made directly over the adenoma under ultrasound guidance. No flap is raised as it is done for 
the four-gland exploration, but the strap muscles are split to gain direct access to the parathyroid adenoma. The adenoma is then identified and excised being constantly aware of the important relationship of the parathyroid with the recurrent laryngeal nerve (RLN) and the inferior thyroid artery.

In this study, we are presenting the focused parathyroidectomy under sonar guidance indicated for parathyroid adenoma. The procedure is limited to the cases where pre-operative ultrasound and Sestamibi are concordant. Ultrasound is more subjective, but in experienced hands, it adds more value to the preoperative localization and facilitates intraoperative identification of an enlarged parathyroid. The move toward focused parathyroidectomy is also due to the anticipated benefit of potentially fewer complications (RLN injury, hypocalcaemia) associated with unilateral exploration, avoidance of general anesthesia (GA) and with the option of day case surgery. 3,6

In view of the reported benefits of minimal access, we have routinely adopted the focused parathyroidectomy since 2013 where applicable, and we would like to reflect on the result thereof.

\section{Objective}

To reflect on the result of sonar-guided focused parathyroidectomy under superficial cervical block (SCB) advocated for parathyroid adenoma.

\section{Patients and Methods}

Retrospective review of parathyroidectomies performed between January 2013 and December 2014 at Chris Hani Baragwanath Academic Hospital (CHBAH). Data included demographics, localization studies (the ultrasound was performed by a single experienced sonographer), histopathology report, intraoperative findings, type of anesthesia, ICU admission, pre- and postoperative parathormone $(\mathrm{PTH})$ and calcium $\left(\mathrm{Ca}^{+2}\right)$ results. The endocrinologists worked up all patients and there was no case associated with multiple endocrine neoplasia (MEN). All cases done under general anesthesia (GA) or associated with concomitant thyroidectomy were excluded. Ethics approval was obtained from the Human Ethics Committee of the University of the Witwatersrand and the Research Review Board of CHBAH (M150274).

\section{Statistical analysis.}

Descriptive study. Continuous data are presented as mean or median and categorical data are presented as proportion by ratio or percentage.

\section{Anesthesia: Superficial cervical block.}

Patients were counseled for a superficial cervical block and supplementation with local anesthetic if need be. A sterile field was created and patient positioned supine with the neck facing the opposite side. A 22-gauge needle was inserted perpendicular to the skin in the middle of the posterior border of the sternocleidomastoid muscle (SCM). After feeling a "pop", $10 \mathrm{ml}$ of $0.5 \%$ plain macaine was injected. Additional $4 \mathrm{ml}$ of macaine was used to fan caudally and cranially. Vitals (BP, HR, oxygen saturation) were monitored throughout the procedure.

\section{Ultrasound}

A high-end ultrasound machine, Aloka Prosound, SSD alpha 10 with color Doppler imaging display was used. A linear compound array transducer with a frequency range of 10 to $14 \mathrm{MHZ}$ was the preferred transducer of choice in achieving optimal spatial resolution. ${ }^{12}$

The patients were placed in a supine position with the neck slightly hyperextended. A soft pad, sponge or rolled towel was placed under the patient's shoulders to maintain the hyperextension of the neck and to lift the inferior pole of the thyroid out of the mediastinum. ${ }^{13}$ Patients were scanned preoperatively during the diagnosis work-up and again intraoperatively by a single dedicated sonographer.

The ultrasound evaluation started with the survey of the entire thyroid gland using gray scale imaging to rule out intrathyroidal parathyroid adenoma. ${ }^{14}$ The color and power Doppler imaging displays were used to supplement gray scale imaging to demonstrate vascularity within the adenoma. The parathyroid was recognised by its peripheral feeding vessel and a typical arc or rim vascularity. ${ }^{15}$ The majority of adenomas are homogeneously hypoechoic from the thyroid gland, ${ }^{15}$ and may contain cystic areas that represent degeneration or in rare cases may be completely cystic, these are true cysts. The images included the superior, mid and inferior portions of the thyroid lobes bilaterally as well as the upper mediastinum. ${ }^{14}$

Although the normal parathyroid glands are usually not visualised with available sonographic technology, enlarged parathyroid glands may be visualised. ${ }^{16}$

A parathyroid gland is considered to be enlarged if it measures greater than 5-7 mm long, 1-2 $\mathrm{mm}$ thick and 3-4 mm wide. ${ }^{12}$

\section{9m Tc-Sestamibi scan}

Patients were injected with $740 \mathrm{MBq}$ of $99 \mathrm{~m} \mathrm{Tc}-$ Sestamibi. The images were acquired on a double head gamma camera equipped with CT (GE Hawkeye and Philips Bright view $\mathrm{XCT})$.

Dynamic images over the anterior neck were acquired for 3 minutes in a $128 \times 128$ matrix at 2 seconds per frame. Planar images of the neck and mediastinum were then acquired at 5, 20, 60 and 180 minutes post tracer injection. The anterior images were acquired for 3 minutes in a $128 \times 128$ matrix with a $20 \%$ window centered over the $140 \mathrm{keV}$ photo peak, using a LEHR collimator. SPECT/CT image acquisition was performed at 25 to 30 minutes, immediately after the 20 minutes planar image, with the field of view including the neck and mediastinum. A matrix of $64 \times 64$ was used for the SPECT data acquisition with 120 projections over a $360^{\circ}$ arc, with 30 seconds per projection. 
Two experienced nuclear physicians reviewed the images. Areas of focal increased tracer uptake in the neck or mediastinum on the early images that demonstrated retention or further increased uptake on the delayed images were considered as positive for the presence of hyperfunctioning parathyroid glands, likely adenoma. The location of the suspected parathyroid adenoma was further classified as left or right sided, superior, inferior or ectopic. The findings were considered negative if no areas of focal tracer uptake were seen in the neck or mediastinum, on the early and the delayed images. ${ }^{17-24}$

\section{Results}

A total of 20 patients had a parathyroidectomy between January 2013 and December 2014. Five patients were excluded because they had either a GA or associated thyroidectomy. The remaining 15 patients underwent focused parathyroidectomy under SCB. The mean age was 61.4 (45-85), majority of patients were female (13/15) with a female to male ratio of $6.5: 1$. The median hospital stay was one day; one patient stayed for 6 days due to the underlying comorbidities. There was no high care admission. The majority of adenomas $(86.6 \%, 13 / 15)$ were located on the inferior pole of the thyroid and there was equal distribution between left and right sides (7 vs. 8). The Sestamibi reported one intrathyroidal adenoma that was proven to be extrathyroidal on ultrasound and surgical exploration. Hypercalcaemia resolved in all patients. One patient developed persistent hypocalcaemia beyond three months. There was one temporary left recurrent laryngeal nerve palsy that resolved after 12 months and no mortality. There was no conversion to GA and no procedurerelated complication associated with the use of SCB. The number of patients in American Society of Anesthesiology (ASA) classified as I, II and III was 2,10 and 3 respectively.

\section{Discussion}

There was a good correlation between localising studies (Sestamibi, ultrasound), intraoperative findings, histopathology report and postoperative blood result (calcium and $\mathrm{PTH}$ ).

\section{Intraoperative $\mathrm{PTH}$ rapid essay}

One of the prerequisites for MIP is the availability of a quick PTH essay. This test is not available at our institution. However, the lack of this did not adversely affect our result. A possible explanation is the rarity of bilateral adenoma in sporadic primary hyperparathyroidism in our study group ${ }^{1}$ on one hand and the concordant preoperative localizing imaging that reduced the risk of error on the other hand.

\section{Ultrasound}

Ultrasound is an excellent non-invasive, inexpensive and readily available imaging modality that is helpful in the evaluation of parathyroid adenoma. The success depends on the use of a high-resolution machine and high frequency linear transducer. Ultrasound was used to substantiate the Sestamibi findings and, where concordant, to guide the minimal access surgery.

The operator, the quality of ultrasound machine, and the abnormal site of the parathyroid determine the shortcoming of ultrasound. A tracheo-esophageal groove, mediastinal or retroesophageal parathyroid adenoma makes ultrasound localization poor if not impossible because of the acoustic shadow cast by the larynx, trachea and the sternum. Likewise

\begin{tabular}{|c|c|c|c|c|c|c|c|}
\hline No & $\begin{array}{c}\text { Age, } \\
\text { gender }\end{array}$ & $\begin{array}{l}\text { Sestamibi, sonar } \\
\text { surgical location }\end{array}$ & Histology & $\begin{array}{c}\text { Preop Ca } \\
\mathrm{Mmol} / \mathrm{L} \\
\end{array}$ & Preop PTH & Postop PTH & $\begin{array}{c}\text { Postop } \mathrm{Ca}^{++} \\
\text {within } 3 \text { months }\end{array}$ \\
\hline 1 & $54 \mathrm{~F}$ & LS & $\mathrm{A}$ & 3.32 & 17 & - & 1.66 \\
\hline 2 & $65 \mathrm{~F}$ & LI & A & 2.9 & 17 & - & 2.35 \\
\hline 3 & $69 \mathrm{~F}$ & LI & A & 2.9 & 14.7 & - & 2.25 \\
\hline 4 & $45 \mathrm{~F}$ & LI & A & 2.74 & 11.1 & - & 2.44 \\
\hline 5 & $52 \mathrm{M}$ & RI & A & 3.33 & 16 & - & 2.09 \\
\hline 6 & $77 \mathrm{~F}$ & RI & A & 2.94 & 43.3 & 3.3 & 2.43 \\
\hline 7 & $85 \mathrm{~F}$ & RI & A & 2.94 & 12.3 & - & 2.33 \\
\hline 8 & $62 \mathrm{M}$ & RI & A & 2.99 & 21 & 0.6 & 2.31 \\
\hline 9 & $62 \mathrm{~F}$ & LI & A & 2.66 & 10.1 & 5.8 & 2.39 \\
\hline 10 & $49 \mathrm{~F}$ & RI & A & 3.1 & 30 & 1.6 & 2.07 \\
\hline 11 & $60 \mathrm{~F}$ & LS & A & 3.05 & 24.4 & 1.2 & 2.27 \\
\hline 12 & $65 \mathrm{~F}$ & RI & A & 3.02 & 32.6 & 1.7 & 2.37 \\
\hline 13 & $65 \mathrm{~F}$ & RI & A & 2.7 & 9.8 & 4.8 & 2.29 \\
\hline $14 \#$ & $53 \mathrm{~F}$ & LI & A & 3.31 & 21 & 3.5 & 2.23 \\
\hline 15 & $58 \mathrm{~F}$ & RI & A & 2.62 & 11.8 & 4.9 & 2.30 \\
\hline
\end{tabular}


an ectopic parathyroid will not be detected. The fact that a sonographer devoted to the parathyroid localization did all ultrasounds may explain the good correlation between the more objective Sestamibi and the more subjective ultrasound.

With the routine use of sonar guided focused parathyroidectomy under SCB, high care observation postoperatively was no longer mandatory in our institution.

\section{Pitfall of ${ }^{99 m}$ Tc-Sestamibi}

The ${ }^{99 \mathrm{~m}} \mathrm{Tc}-$ Sestamibi inaccurately reported one intrathyroidal adenoma despite the use of SPECT (single-photon emission computed tomography) that provides three-dimensional images for better localization of parathyroid adenoma. This happens when the parathyroid gland is very close to the thyroid and may even be attached to or covered by the thyroid capsule. Because of to the limited resolution of the gamma camera, these parathyroid glands may then falsely appear to be intrathyroidal on the ${ }^{99 \mathrm{~m}} \mathrm{Tc}-$ Sestamibi images.

\section{Superficial cervical block}

SCB was well tolerated. Occasionally the surgeon had to add local anesthesia when patients experienced pain despite the block. Complications are rare and include injection into the dural sac creating an epidural or spinal block, injection into vertebral artery, phrenic nerve blockade, nerve injury, local anesthetic toxicity, haematoma and sepsis. ${ }^{25,26} \mathrm{SCB}$ is advantageous especially for patients with multiple comorbidities that are considered high risk for general anesthesia.

\section{Discharge time}

Although the patients could have been discharged the same day, our protocol requires confirmation of normocalcaemia and review by endocrinologists prior to discharge, especially when most of our patients were ASA II and III

\section{Conclusion}

With concordant preoperative Sestamibi and ultrasound localization, sonar guided focused parathyroidectomy was feasible and achieved correct identification of the adenoma that was confirmed histologically and biochemically. SCB was successful in all cases and obviated the need for GA and high care.

\section{REFERENCES}

1. William B, Inabnet $\mathrm{J}$, Lee $\mathrm{A}$, et al. Parathyroid disease. In Garden JO, Paterson-Brown S. $4^{\text {th }}$ Edition. Endocrine Surgery. Saunders. Elsevier. 2009:1-17.

2. O'Doherty MJ, Kettle AG, Wells P, et al. Parathyroid imaging with technetium-99m-sestamibi: preoperative localization and tissue uptake studies. J Nucl Med. 1992;33:313-8.

3. Udelsman R, Lin Z; Donovan P. The superiority of minimally invasive parathyroidectomy based on 1650 consecutive patients with primary hyperparathyroidism. Ann Surg. 2011;253(3):58591 (ISSN: 1528-1140).

4. Wong W, Foo FJ, Lau MI, Sarin A, Kiruparan P. Simplified minimally invasive parathyroidectomy: a series of 100 cases and review of the literature. Ann R Coll Surg Engl. 2011;93(4):2903 (ISSN: 1478-7083)

5. Schneider DF, Mazeh H, Sippel RS, et al. Is minimally invasive parathyroidectomy associated with greater recurrence compared to bilateral exploration? Analysis of more than 1,000 cases. Surg. 2012;152(6):1008-15 (ISSN: 1532-7361).

6. Gracie D, Hussain SS. Use of minimally invasive parathyroidectomytechniques in sporadic primary hyperparathyroidism: systematic review. J Laryngol Otol. 2012;126(3):221-7 (ISSN: 1748-5460).

7. Karahan Ö, Okus A, Sevinç B, et al. Minimally invasive parathyroidectomy under local anesthesia. J Postgrad Med. 2013;59(1):21-4 (ISSN: 0022-3859).

8. Melck AL, Armstrong MJ, Yip L et al. Case-controlled comparison of video-assisted and conventional minimally invasive parathyroidectomy. Am Surg. 2012;78(1):12532 (ISSN: 1555-9823).

9. Venkat R, Kouniavsky G, Tufano RP, et al. Long-term outcome in patients with primary hyperparathyroidism who underwent minimally invasive parathyroidectomy. World J Surg. 2012;36(1):55-60 (ISSN: 1432-2323).

10. Versnick M, Popadich A, Sidhu S, et al. Minimally invasive parathyroidectomy provides a conservative surgical option for multiple endocrine neoplasia type 1-primary hyperparathyroidism. Surg. 2013;154(1):101-5 (ISSN: 15327361).

11. Day KM, Elsayed M, Monchik JM. No need to abandon focused unilateral exploration for primary hyperparathyroidism with intraoperative monitoring of intact parathyroid hormone. J Am Coll Surg. 2015;221:518-23. (ISSN1072-7515/15)

12. Tempkin BB. (2007). Ultrasound scanning principles and protocols. $2^{\text {nd }}$ edition, Philadelphia: Saunders.

13. Khan AR, Ellemdin NS, Gayaparsad, K. (2008). Ultrasoundfirst modality in the detection of parathyroid adenomas. S Afr J Radiology.

14. AIUM guidelines

15. Wieneke JA \& Smith A. (2008). Parathyroid adenoma. Head Neck Pathol. 2(4):305-8.

16. Rumack CM \& Wilson S.(2011). Diagnostic ultrasound, vol 2, fourth edition by Mosby,Inc.

17. Erbil Y, Barbaros U, Yanik B, et al. Impact of gland morphology and concomitant thyroid nodules on preoperative localization of parathyroid adenomas. Laryngoscope. 2006;116:580-5.

18. Taieb D, Hindie E, Grassetto G, et al. Parathyroid scintigraphy When, How and Why? A concise systematic review. Clin Nuc Med. 2012;37:568-74.

19. Pata G, Casella C, Besuzio S, et al. Clinical appraisal of $99 \mathrm{~m}$ technetium-sestamibi SPECT/CT compared to conventional SPECT in patients with primary hyperparathyroidism and concomitant nodular goiter. Thyroid. 2010;20:1121-7.

20. Erbil Y, Barbaros U, Tukenmez M, et al. Impact of adenoma weight and ectopic location of parathyroid adenoma on localization study results. World J Surg. 2008;32:566-71.

21. Nichols KJ, Tomas MB, Tronco GG, et al. Sestamibi parathyroid scintigraphy in multigland disease. Nucl Med Commun. 2011;33:43-50

22. Erbil Y, Kapran Y, Issever H, et al. The positive effect of adenoma weight and oxyphil cell content on preoperative localization with $99 \mathrm{mTc}$-sestamibi scanning for primary hyperparathyroidism. Am J Surg. 2008;195:34-9.

23. Shiau YC, Tsai SC, Wang JJ, et al. Detecting parathyroid adenoma using technetium-99m tetrofosmin: comparison with P-glycoprotein and multidrug resistance related protein expression - a preliminary report. Nucl Med Biol. 2002;29:33944.

24. Ciappuccini R, Morera J, Pascal P, et al. Dual-phase ${ }^{99 m} \mathrm{Tc}$ Sestamibi scintigraphy with neck and thorax SPECT/CT in primary hyperparathyroidism. Clin Nucl Med. 2012;37:223-8.

25. Kanthan RK. The use of superficial cervical plexus block in oral and maxillofacial surgical practice as an alternative to general anesthesia in selective cases. Ann Maxillofac Surg. 2016;6(1):48. Doi: $10.4103 / 2231-0746.186120$

26. NYSORA 4-Part. Online CME webinar series 